\title{
Invasive Illness with Salmonella virchow Infection
}

\author{
V. MANI, J. BRENNAND, B. K. MANDAL
}

British Medical fournal, 1974, 2, 143-144

Salmonella virchow infection in man is usually sporadic and is little known in Britain. We report an outbreak of 21 cases in the Manchester area. The source of infection was believed to be chickens bought at different shops. Symptoms were typhoidal or septicaemic.

\section{Introduction}

Though sporadic cases of Salmonella virchow infection in man have been reported from widely scattered parts of the world for many years it is a relative newcomer in Britain as an impontant cause of human infection. The annual average of 11 identifications between 1962-6 in England and Wales rose to 51, 299, and 361 in 1967, 1968, and 1969 respectively, after which there was a drop again with annual totals of 96,95 , and 80 , in 1970,1971, and 1972 respectively (Rowe, 1973). Poultry, particularly chicken, have been the chief source of infection and in 1968 there was a large outbreak in the Liverpool area, the source being broiler chicken from a particular packing station (Semple et al., 1968). In the summer of 1972 there was an interesting outbreak in Manchester, where an unusually high proportion of cases had typhoidal or septicaemic features. Our paper deals with this outbreak and thus draws attention to a hitherto unreported aspect of $S$. virchow infection.

\section{Outbreak}

Over a 14-day period in July and August 1972 six patients with pyrexia of unknown origin, admitted to the Regional Infectious Diseases Department at Monsall Hospital, Manchester, were found to be suffering from $S$. virchow infection, thus bringing the outbreak to light. There had been no isolation of this serotype during the previous five months either from the numerous cases of gastroenteritis at this hospital or by the public health laboratory from cases arising in the community. Detailed bacteriological screening among the contacts uncovered 14 more cases, none of whom had typhoid-like symptoms, and we learnt of another patient with $S$. virchow septicaemia in a general hospital some distance away.

The patients who were admitted to hospital were all unrelated and came from widely different parts of the city and its suburbs, and though chicken was strongly suspected as the source of infection this was never proved. All the patients had recently eaten chicken, which in most cases was bought cooked but in one case was bought raw. The chickens were bought from different shops. Despite intensive swabbing of the premises and examination of chicken samples all tests were negative. The outbreak ended as abruptly as it had started and during the rest of that year no further cases came to light either in this hospital or in the community.

\footnotetext{
Department of Infectious Diseases and Bacteriology, Monsall Hospital, Manchester M10 8WR

V. MANI, M.B., M.R.C.P., Senior Registrar

J. BRENNAND, F.I.M.L.T., Chief Technician

B. K. MANDAL, M.B., M.R.C.P., Consultant Physician
}

\section{Case Reports}

Case 1.-This 2-year-old boy was admitted to hospital with a fiveday history of illness, his chief symp+oms being lack of appetite and fever and, later, diarrhoea and vomiting. He was toxic, feverish, and had a distended, tympanitic abdom.n. He had watery diarrhoea, his stools averaging from six to 10 a day. The pvrexia continued for four days, temperature varying from 38 to $40.8^{\circ} \mathrm{C}$, and his condition deteriorated gradually, needing intravenous fluid therapy. The blood and stool cultures both grow $S$. virchow. Chloramphenicol treatment was begun on the third day after admission, on receipt of the blood culture report. Defervescence was evident within 48 hours though the abdominal distension and diarrhoea took a further two days to settle. He was discharged home a week later as a convalescent carrier.

Case 2.-This 10-year-old boy was admitted to a nearby hospital with a history of fever for five days and convulsions for one day. He also suffered from abdominal pains. He was toxic and feverish on admission and remained so for another three days, during which period he developed diarrhoea. His blood culture was sterile but stools grew $S$. virchow. When transferred to our care he had pyrexia and a generally tender, tympanitic abdomen. His condition settled down after a few days without treatment and at the time of discharge he was still excreting the organism. The only medication he had received was anticonvulsants from his doctor before hospitalization.

Case 3.-This 13-year-old boy was admitted with a five-day history of abdominal pain, fever, and vomiting, which all appeared suddenly at about the same time. He was toxic and feverish (body temperature $40.2^{\circ} \mathrm{C}$ ). The abdomen was distended and slightly tender, the liver and spleen were slightly enlarged. There were no other abnormal findings on physical examination. $S$. virchow was grown from blood and stool cultures. The results of liver function tests were normal. He continued to have pyrexia, his temperature ranging between $38^{\circ}$ and $40^{\circ} \mathrm{C}$. His stools were loose and numbered up to two a day. The fever responded to chloramphenicol within 24 hours. He was discharged home 10 days later, symptom free but still excreting the organism.

Case 4.- This 22-year-old woman had an illness which began with anorexia after a bout of gastroenteritis seven days before admission. Though her bowel symptoms had subsided within 36 hours she had generally remained unwell, with fever, headaches, nausea, and abdominal discomfort. On admission she was feverish but, apart from minimal meningism, she had no physical signs. The next day two rose spots were noted on her anterior abdominal wall. She continued to have fever, the temperature varying between $38^{\circ}$ and $40^{\circ} \mathrm{C}$. Her cerebrospinal fluid was normal but blood cultures grew $S$. virchow. The pyrexia responded to chloramphenicol, which was continued for 10 days. She was still excreting the organism at the time of her discharge from hospital.

Case 5.-This 8-year-old girl was admitted with a five-day history of pyrexia and vomiting which had failed to respond to penicillin. She had had no diarrhoea at any time. When admitted she was toxic, with a temperature of $39.5^{\circ} \mathrm{C}$. She had a fullness of the abdomen with tenderness around the umbilicus. All other systems were clinically normal. Investigations on admission included a blood culture, which grew $S$. virchow. The stools were repeatedly positive for the same organism. She continued to be ill, her temperature ranging between $37.5^{\circ}$ and $39.5^{\circ} \mathrm{C}$. Chloramphenicol treatment begun when the result of the blood culture was received and she soon began to improve. She was, however, still excreting the organism at the time of her discharge.

Case 6.- This 25-year-old man was admitted with a five-day history of fever, malaise, and abdominal pains and a three-day history of diarrhoea and vomiting. He had been treated with ampicillin by his doctor with no improvement. On examination he was toxic and pyrexial but there were no other abnormalities. Blood and stool cultures grew $S$. virchow. Chloramphenicol was given when the blood culture report was received two days after admission, and after 24 hours the temperature reverted to normal. 
Unfortunately the patient discharged himself against medical advice before completing his treatment.

Case 7.-This 59-year-old man was admitted to a local hospital with a two-week history of anorexia and watery diarrhoea with stools numbering up to 12 a day. He had first consulted his doctor two days after he fell ill and was prescribed ampicillin, which he had been taking until his hospital admission. He had developed haematuria four days before and also had been coughing up blood for four days. On admission he was drowsy, hypotensive, apvrexial, and hyperventilating. The abdomen was generally tender; the left kidney was palpable. The blood urea was high and the findings were suggestive of renal failure. While undergoing peritoneal dialysis he collapsed and died. Culture of blood taken on admission grew $S$. virchow. Necropsy showed severe benign nephrosclerosis, renal calculi and hydronephrosis of the left kidney, cortical hyperplasia of the left adrenal gland, and extensive myocardial infarction. There was little doubt that invasive $S$. virchow infection, leading to dehydration and hypotension, had been a major contributory factor in causing the death of this patient with pre-existing severe renal and myocardial disease.

\section{OTHER CASES}

Of the 14 other cases who were not admitted to hospital six were members of the family of the patient in case 1, of whom all except one had mild to moderate diarrhoea. Another five cases were detected among the members of a family whose source of chicken was the same as that of the family of the patient in case 1 . Of these five only one was asymptomatic and the others suffered from diarrhoea. Three relatives of the patient in case 3 also had mild diarrhoea and positive stool culture. No evidence of infection could be found among members of the family of the other five patients admitted to hospital.

Thus the clinical illness in seven of the 21 cases in the outbreak was dominated by typhoidal or septicaemic features such as pronounced fever, toxic state, and distended abdomen. Diarrhoea was either absent or mild in all except cases 1 and 7. In all cases apart from case 2 the blood cultures were positive, and despite the negative blood culture the clinical illness in case 2 was characterized by persistent fever and a toxic state, justifying its inclusion in this group.

\section{Discussion}

The presence of typhoidal or septicaemic features in all seven of the hospital patients, comprising a third of the total number of cases in the outbreak, is of considerable interest. Generally speaking salmonella serotypes other than $S$. typhi and $S$. paratyphi remain confined to the gastrointestinal tract, acute diarrhoeal illness being the commonest clinical expression. Though typhoidal or septicaemic features are by no means rare they are not constant except in infections with $S$. cholerae-suis and $S$. sendai. In a large series of $S$. choleraesuis infections reponted by Saphra and Winter (1957) $47.6 \%$ had a typhoidal or septicaemic syndrome. Infection with this serotype, however, is quite rare in this country, with only eight cases isolated in England and Wales during 1970 and 1971 (Rowe, 1973).

Our cases suggest that $S$. virchow is yet another serotype with a high invasive propensity. This characteristic has not, however, been previously emphasized, though it is relevant that of the 35 cases of $S$. virchow infections admitted to hospital in the Liverpool outbreak (Semple et al., 1968) blood cultures were taken in 2? cases and no fewer than eight had positive cultures. The ratio of septicaemic cases to the total number of cases in the outbreak was, however, much lower. In our own hospital based series, which was naturally biased in favour of more severe cases, $39(10 \%)$ of the 394 cases of salmonella infection admitted during a four-year period (196972) had positive blood cultures and 18 of these were from 43 typhoid or paratyphoid cases $(42 \%)$. Out of 303 cases of salmonellosis due to all other serotypes (but excluding $S$. virchow) 12 had positive blood cultures, giving a septicaemia rate of $4 \%$. Before the 1972 outbreak in Manchester described in this paper $S$. virchow was isolated on four occasions from 42 patients $(10 \%)$. Thus, the high incidence of septicaemia noted in the Manchester 1972 outbreak was not present in these earlier figures. An alternative explanation of the present outbreak could therefore be that it was due to a single strain (clone) of $S$. virchow and that this clone may have had a special invasive propensity.

There seems no doubt, however, that $S$. virchow can be unusually invasive at times, presenting as an essentially typhoidal illness with little or no bowel disturbance. Future outbreaks will be observed with close interest to see how often this mode of presentation occurs.

We thank Dr. B. Rowe, director of the salmonella and shigella reference laboratory, Central Public Health Laboratory, Colindale, London, for serotyping and for his helpful comments; Dr. J. D. Abbott, Public Health Laboratories, Manchester, for information about the cases not admitted to hospital; Dr. A. G. Ironside, consultant physician at Monsall Hospital, and Dr. Donald Longson, consultant physician at Manchester Royal Infirmary, for permission to publish cases under their care; and Dr. R. F. Williams, consultant microbiologist at Monsall Hospital, for his help with the Monsall Hospital data.

A modified version of this paper was presented at the International Conference on Antimicrobial Agents and Chemotherapy at Washington D.C. on 21 September 1973.

\section{References}

Rowe, B. (1973). Personal communication.

Saphra, I., and Winter, J. W. (1957). The New England fournal of Medicine, 256, 1128.

Semple, A. B., Turner, G. C., and Lowry, D. M. O. (1968). British Medical fournal, 4, 801 . 